\title{
A dynamic cruise control system for effective navigation system
}

\author{
T. Someswari ${ }^{1}$, Anil Kumar Tiwari $^{2}$, Nagraj R. ${ }^{3}$ \\ ${ }^{1}$ Department of Electrical and Electronics Engineering, The Oxford College of Engineering, India \\ ${ }^{2}$ Amity School of Engineering, India \\ ${ }^{3}$ Kalasalingam University, India
}

\begin{tabular}{l} 
Article Info \\
\hline Article history: \\
Received Jul 27, 2019 \\
Revised Feb 29, 2020 \\
Accepted Mar 21, 2020 \\
\hline
\end{tabular}

\section{Keywords:}

Computational complexity

Fuzzy

GPS

INS

Navigation

Sliding mode controller

Switching action

Tracking error

\begin{abstract}
With the fast development of artificial intelligence, robotics, and embedded system along with sensor technologies, the speed control mechanism is required in various other applications such as automatic or self-piloting aircraft, auto-driven vehicles, auto driven lifts and much other robotics based automation plants, etc. For each unpredictable and progressed vehicular framework accompanies a better route that is fit for utilizing the two GPS and INS related sign. There have been a noteworthy number of research works being completed towards creating sliding mode control framework. In case of inaccurate navigational data or no availability of navigational service, the cruise control could also stop working. Hence, there is a need to evolve up with a novel system offering reliable and fault tolerant navigation system in order to minimize the dependencies on GPS-based information and maximize the utilization of INS based information. This manuscript presents a dynamic cruise control system to achieve better navigation under uncertainties. The performance of the system is analyzed by incorporating sliding mode and fuzzy logic and achieves better accuracy in tracking error, computational complexity ( $28 \mathrm{sec}$ of simulation time) under chattering and switching action operation.
\end{abstract}

Copyright (C) 2020 Institute of Advanced Engineering and Science. All rights reserved.

\section{Corresponding Author:}

T. Someswari,

Department of Electrical and Electronics Engineering,

The Oxford College of Engineering,

10th Milestone, Bommanahalli, Hosur Road, Bengaluru, Karnataka 560068, India.

Email: someswarioxford@gmail.com

\section{INTRODUCTION}

The growing progression in communication mechanism has witnessed a proportionate increment in a navigation offering devices [1,2]. Nowadays, both the inertial navigation system (INS) and global positioning system (GPS) were used for a better form of navigation system [3, 4]. From the various existing researches, it has been observed that significant quantities of research work have concentrated on joint usage of INS and GPS. Critical research suggests that the navigational data offered by the INS framework exhibits some of the issues related to the navigational system [5]. Even though these issues in navigational data of INS are of short duration, but it precipitously degrades after some time. Then again, navigational data offered by GPS is viewed as profoundly stable alongside a better arrangement of exact information identified with speed, area, and disposition [6]. Also, the identified information with all these physical and kinetic substances are given by INS while GPS provides data identified with just area and speed. There is additionally a potential reliance of the two INS as GPS together as a successful and dependable navigational data can't be offered by INS independent, and the principle purpose for it is more occurrences of issues related with inertial sensors. It has likewise been seen that INS can provide a constant stream of navigational information while information stream is always discontinuous for GPS inferable from different types of sign impedance causing blackout $[7,8]$. Also, $95 \%$ of the current research-based work has thought about the two 
INS and GPS for developing up with a superior type of navigational framework. Use of such joint navigational framework can be seen in various types of vehicle proceeding onward land [9], water [10], and air [11]. There are additionally different types of weapons like rockets uses such joint navigational framework [12]. It is observed that there has been the great number of research work completed in this respects, and yet, it can't be overlooked that current research methodologies were not appeared to effectively benchmark or professed to offer unrivalled navigational framework [13]. Existing specialists are additionally endeavoring to build cost-effective INS-GPS navigational framework where the conspicuous test is to correctly introduce the outside edge inferable from a corrupted working procedure of gyros or comparable types of different sensors. One of the powerful instruments to address this issue could be by utilizing a sliding mode controller (SMC) [14], which is the core of each development control framework. For each unpredictable and progressed vehicular framework accompanies a better route that is fit for utilizing the two GPS and INS related sign. The recent research of [15] has provided the idea of SMC concept for a robot manipulator.

There has been a noteworthy number of research work being completed towards creating sliding mode control framework; be that as it may, the lion's share of them are related with certain inalienable issues of managing dynamic framework. It was seen that current methodologies don't provide food up to the vulnerability requests of the dynamic framework, particularly in transmission arrange. With dominant part of the work lacking benchmarking and exclusion of considering the contextual analysis of taking care of the navigational issue, the SMC has turned out to be one of the less investigated arrangements toward upgrading route framework utilizing GPS and INS. Therefore, this manuscript presents a dynamic cruise control system (DCCS) to achieve better navigation performance and computational complexity. The manuscript is categorized with different sections like review of literature in Section 2, problem statement in Section 3, proposed system in Section 4, results and analysis in Section 5, and conclusion in Section 6.

\section{BACKGROUND}

Many research works are presented by concentrating on planning a powerful navigational framework utilizing INS just as GPS, with extraordinary strategies and mechanism. Existing works are progressively centered on to achieve better accuracy, where the investigation driven by Dacheng et al. [16] demonstrated upgraded affectability by limiting Doppler frequency when the navigational arrangement of INS is coordinated with GPS. Reception system of channels is one extraordinary methodology in this examination course. As indicated by Cho et al. [17], an upgraded Kalman channel would improve the navigational exhibition, particularly concentrating on cost-adequacy organization perspective. Arrangement of comparative Kalman channel of broadened type was found in introduced by Duong and Nguyen [18] for a compelling estimation of physical navigational parameters for example frame of mind, speed, area, and so forth. Utilization of Kalman channel was likewise found in work examined by Fang and Gong [19] where the prime goal was to consolidate a prescient plan of an incorporated navigational administration. The investigation was tried over constant sensors mounted on an airplane to find that offers better exactness. There was additionally research work which says that creating a navigational framework for a submerged vehicle is extremely a difficult one. Such research work has been done by Lee et al. [20], which is intended for limiting the blunders in navigational framework, particularly with the GPS information. The work did by Li and Sun [21] has additionally utilized Kalman channel of broadened structure for improving the precision related to navigational information. Introduced by $\mathrm{Wu}$ et al. [22] have concentrated on building up a prescient calculation utilizing a Kalman channel. The examination likewise details a commotion model for remunerating the sign. Yan et al. [23] have additionally done work utilizing Kalman channel.

Contextual analysis of the flying article and its improvement of route information was examined by Nakanishi et al. [24]. The researcher has tended to the issue related to unwavering quality of information from GPS by executing a unified system of sensor combination with a guide of updates got in the offbeat method of correspondence. Contextual investigation of the route of the land vehicle has been considered in introduced by Qin et al. [25], where the researcher has utilized Kalman channel just as standard fuzzy inference framework for limiting blunders in route framework. Another researcher, viz. Sun et al. [26] have completed a test based examination where a GPS INS based route framework. It was additionally discovered that coupling among the navigational parameters noteworthy influence the identification of sign. This issue has been explored by Jamal [27] by correcting the mistakes related to detecting during fast information catch. As indicated by the researcher, tight coupling outcomes are intending to these issues. Use of the neural system was seen in introduced by Jaradat and Hafez [28] where a relapse based methodology was executed for tending to the correspondence delay in route framework. The existing framework has likewise seen examination towards recognizing and tending to flaws in the navigational framework. The work did by Xin et al. [28] has utilized measurable based methodology for deficiency distinguishing proof. Also there are 
different examinations towards improving navigational execution, yet a proficient controller can be obtained utilizing a sliding mode configuration approach. There have been different research-based methodologies towards SMC [29, 30] towards tending to various periods of research-based issues. Elgammal and El-naggar [31] has utilized this methodology for creating dynamic power channel. The comparative course of work has been found in introduced by Fei et al. [32], which observed selection of neural system just as the fuzzy-based sliding methodology was utilized for limiting the gabbing impact. Concentrate towards cost advancement utilizing fuzzy based controller configuration has been displayed by Li et al. [33] where the researcher has ordinarily used interim sort two fuzzy methodologies for tending to postpone in the framework. The exhibition of controller configuration utilizing fuzzy based sliding window was demonstrated to be upgraded considering the contextual investigation of vehicle and driving issues. A work of Shen et al. [34] has utilized a versatile component of building up a controller framework utilizing the same fuzzy put together methodology concerning the highest point of diagram hypothesis. The business locales the issues related with the multi-operator framework. The joining of adaptiveness is likewise found in introduced by Wang and Fei [35] where the disposal of jabbering has been done alongside a versatile element of dependability. Wen et al. [36, 37] have built up a component where the dynamic highlights have been consolidated just as they have likewise centered on creating shortcoming tolerant actuator structure with enough versatile highlights. Another exceptional type of usage has been seen in introduced by Yu et al. [38] where a bio-motivated calculation has been intended for better physical control framework. The work did by Zhao et al. [39] has planned a fuzzy controller framework considering the contextual investigation of shut circle framework related to the multi-input and multi-yield framework. Also, other enhancement based methodologies were discussed with SMC plan, for example, Someswari and Anil Kumar Tiwari [40, 41]. Thus, there have been different files of research works, where the fuzzy-based framework has been observed to be effectively utilized in upgrading the presentation of sliding mode converter and also a number of work being performed towards navigational methodologies too. The next section discusses research problems associated with the existing system.

\section{PROBLEM IDENTIFICATION}

At present, the modern vehicles are equipped with a cruise control system, which is one reduces the effort of manually maintaining the acceleration while driving. However, there is no physical connection between the cruise control device and navigation system. However, with the inclusion of more smart and intelligent components on-vehicle navigation system, it is imperative that the cruise control system could be further integrated with the navigational system. At present, there is not a single research work toward this issue. However, there is a good possibility as there is certain research discussion on using TCP for cruise control. In case of inaccurate navigational data or no availability of navigational service, the cruise control could also stop working. Therefore, the problem statement for the proposed system can be stated as "it is quite challenging to meet as an effective cruise control system must be able to perform proper navigation by understanding the static and dynamic attributes associated with the existing navigational system". The next section briefs about the proposed research methodologies used to address the above set of problems.

\section{THE PROPOSED METHODOLOGY}

The prime motive of proposed novel system is to evolve up offering reliable and fault tolerant navigation system in order to minimize the dependencies on GPS-based information and maximize the utilization of INS based information which has been not much considered in the exsiitngsystem [42-45]. In order to enhance the performance of the navigational system, the introduced study incorporats the variables of the mathematical model with the planar motion of the vehicle. Also, it has been aimed to improvise the fuzzy controller using sliding mode (proposed in [46]) and achieve accurate direction of mobility considering the vehicular characteristics as a constraint. In this model, the fuzzy logic is associated with the formulation of the various rule set to have a better dependency on resources during the navigational process. Also, it has been observed that not many types of research were observed with optimization in the fuzzy processing using SMC. Also, the use of conventional training/learning based algorithm can enhance the dependency of the dataset and is never feasible in online analysis. Also, it has been observed that some of the recent researches have used fuzzy logic type-2 and SMC to address the various issues of the control system. It is found that fuzzy logic type-2 has good potential to handle uncertainty problems but are always associated with computational complexity problems. Thus, the following model is introduced to fulfill the needs of performance enhancement in terms of accuracy and computational efficiency. The design of the proposed system is carried out using analytical research methodology with the following scheme, as shown in Figure 1. 


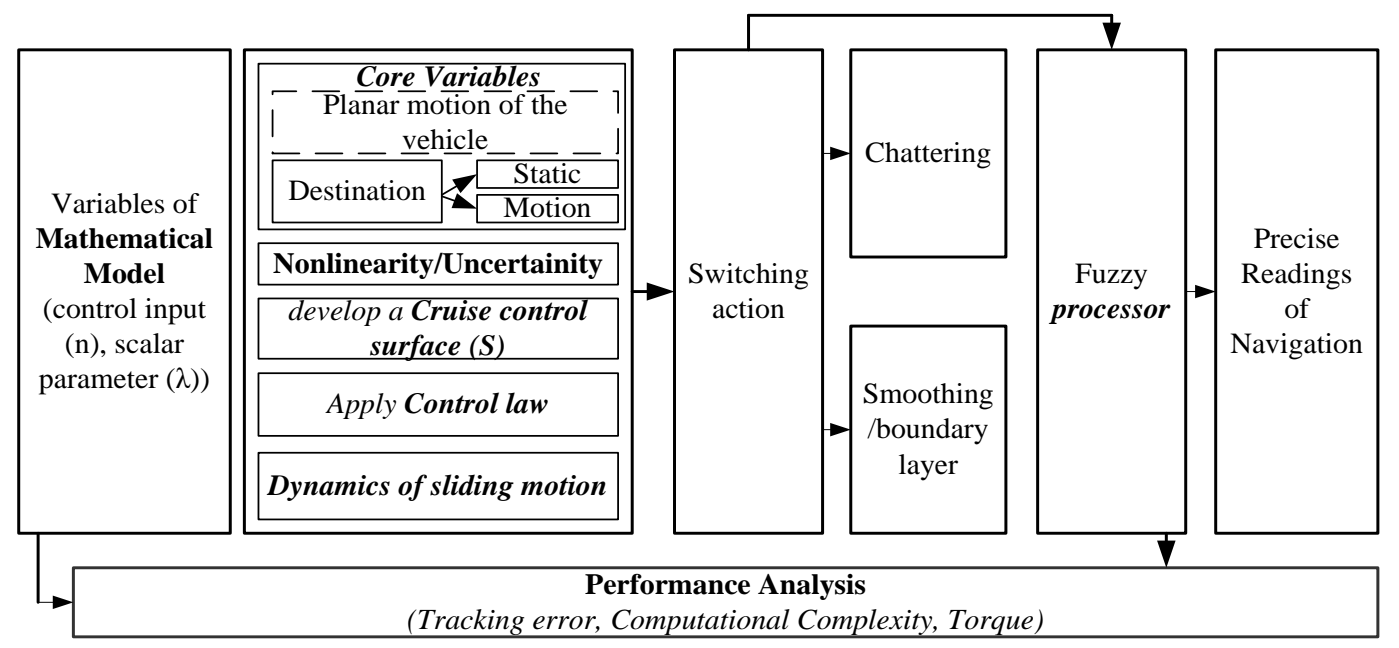

Figure 1. Architecture of the proposed study

\subsection{Model for working performance enhancement}

The proposed study is intended to design a planar motion of the vehicle with the targeted destination. In practical, the destination is always considered to be static (e.g., home, office, park, hospital, etc.); however, there may be a possibility that such destination could also be mobile (e.g., tracking another vehicle). Therefore, the target destination can static or mobile and the system uses INS in this part to feed the input of vehicle speed and the directionality vector of destination. For simpler implementation, both vehicle and (mobile) destination have considered approximately equal speed. This incorporates the most challenging scenario in navigation system implemented till date. Mathematical modeling is carried out for formulating the vehicle-destination geometry along with consideration of various forms of uncertainty problems surfaced from readings of INS navigational system. The study develops a model which relates to vehicle and destination and extracts various uncertain information which is fed to fuzzy logic and sliding mode control for obtaining accurate navigational information. The proposed system considers various issues in the navigation system (chattering) and incorporated the switching control and smoothing (boundary layer) mechanism.

\subsection{Model for computational efficiency}

This part of the study applies an analytical modeling approach to perform optimization. The complete optimization is based on the concept that SMC offers better compensation plan for mitigating uncertainty problems along with fuzzy logic. However, while doing so, it cannot address the problem if the uncertainty attributes are of fluctuating origin, and in such case, SMC is no longer productive when working with the fuzzy logic system. Therefore, one of the better solutions is to offer optimization. In this case, the surface design of sliding mode is introduced by re-considering the proposed vehicle navigational system that is essentially MEMS-based. The first part of the optimization is meant with determining the parameter of the surface, followed by implying control law by considering state trajectories. Simple mathematical modeling will be carried out towards exploiting the dynamics of sliding motion in order to incorporate more flexibility in design parameters. Another proof of optimization will be investigated in this research stage by minimizing the number of Ruleset to a considerably lower degree. The proposed system incorporates the design of the sliding surface, control law, and computational complexity.

\subsection{System model}

The proposed study has considered that at some condition, the precise system modeling is not possible as the external aspects like frictions, forces affect the stability of the system. As it is observed that there are practical difficulties into modelling a cruise navigation system accurately because of various influencing factors of forces acting a surface including friction and all, which has got significant influence on the stability of the control system. These kinds of highly dynamic and nonlinear systems required a flexible and adaptive dynamic cruise control system. To achieve this fuzzy control mechanism based on sliding mode is adopted.

For system design, the mathematical approach is considered where if the DCCS comes with the dynamics' and control input ' $n$ ' for the control then the system can be defined as: 


$$
(x / t)=m(x, t)+\eta(t)
$$

The proposed DCCS is assumed as of $2^{\text {nd }}$ order and is normalizes as $\vec{x}=m+n$. In order to design a control mechanism for displacement and speed. The dynamic cruise control surface (S) for the DCCS is designed as:

$$
S=\left(\frac{d}{d t}+\lambda\right) \tilde{x}+\lambda \vec{x}
$$

where $\lambda$ is meant with a single scalar parameter which has the main impact on the cruise control surface (S). The change in $\mathrm{S}$ at $\mathrm{S}=0$, and $\bar{x}=m+n$ is resolved as:

$$
\begin{aligned}
& \vec{S}=0 \\
& \vec{x}-\bar{x}_{d}+\lambda \tilde{x}=0 \\
& m+n-\vec{x}_{d}+\lambda \vec{x}=0 \\
& n=-m+\vec{x} d-\lambda \vec{x}
\end{aligned}
$$

The value of the scalar parameter $(\lambda)$ with a positive parameter is almost arbitrary, and it reduces the dynamics of the system during sliding.

The DCCS model (m) is an approximation of the DCCS model. The approximated control law can be normalized as $\hat{n}=-\widehat{m}+\hat{x} d-\lambda \hat{x}$. Figure 2 shows a geometrical view of the DCCS if it is slide on the sliding surface with slope only $\theta$.

In order to maintain the working of the system in a sliding surface, a switching action is introduced. The switching action $\left(A_{s w}\right)$ can be defined as:

$$
A_{s w}=-A \times \operatorname{sign}(S)
$$

where A is a large positive constant, and its value is selected if $A=\left\{\begin{array}{c}-A @ S>0 \\ A @ S<0\end{array}\right.$. This switching control is discontinuous across the cruise control surface $(S)=0$.

During steady state, the variable "A" commute at higher frequency lies between $A=A$ and $A=-A$. The control law is considered between these low and high-frequency control. As a result, the discontinuous high-frequency control of switching action is appropriate in the navigation system, but it leads to chattering mechanism in control switching. The chattering is the harmful mechanism which leads to low control accuracy and high heat losses in the power circuits. Figure 3 indicates the Chattering phenomenon in the control system.

In order to solve the chattering problem, smoothing is performed over a chattering phenomenon where a smooth approximation (Sap) is replaced in place of the discontinuous sign. The plot in Figure 4 indicates the smoothing in the chattering phenomenon, i.e.:

$$
\begin{aligned}
& A_{s w}=-A \times S_{a p}(S) \\
& S_{a p}==\left\{\begin{array}{cc}
-1 & @ S / \phi<0 \\
0 & @ S / \phi<1 \\
1 & @ S / \phi<-1
\end{array}\right.
\end{aligned}
$$

where $\phi$ indicates the thickness of the boundary layer.

The mathematical model of DCCS is derived from the fuzzy-based sliding mode by addressing the above-stated problems in the dynamic modelling. Figure 5 gives the 2-link system of controlling arm having robot links 1 and 2. The model includes displacements $\theta_{1}$ and $\theta_{2}$ with the length of links $d_{1}$ and $d_{2}$. The mass of every link is represented as $M_{1}$ and $M_{2}$. During this mathematical modelling, Lagrange-Euler formation is used with the following dynamic equation.

$$
\begin{aligned}
& M(\phi) \ddot{\phi}_{d}+F(\phi, \ddot{\phi}) \ddot{\phi}_{d}+G(\phi)=\tau \\
& {\left[\begin{array}{ll}
N_{11} & N_{12} \\
N_{12} & N_{22}
\end{array}\right] \times\left[\begin{array}{l}
\ddot{\phi}_{1} \\
\ddot{\phi}_{2}
\end{array}\right]+\left[\begin{array}{cc}
-F_{12} \ddot{\phi}_{2} & -F_{12}\left(\ddot{\phi}_{1}+\ddot{\phi}_{2}\right) \\
F_{12} \ddot{\phi}_{1} & 0
\end{array}\right] \times\left[\begin{array}{l}
\ddot{\phi}_{1} \\
\ddot{\phi}_{2}
\end{array}\right]+\left[\begin{array}{l}
G_{1} g \\
G_{2} g
\end{array}\right]=\left[\begin{array}{l}
A_{1} \\
A_{2}
\end{array}\right]}
\end{aligned}
$$


where,

$N_{11}=\left(M+M_{2}\right) \times d_{1}{ }^{2}+M_{2} \times d_{2}{ }^{2}+M_{2} \times d_{1} \times d_{2} \times \cos \phi_{2}$

$N_{12}=M_{2} \times d_{2}^{2}+M_{2} \times d_{1} \times d_{2} \times \cos \phi_{2}$

$N_{22}=M_{2} \times d_{2}^{2}$

$F_{12}=M_{2} \times d_{1} \times d_{2} \times \sin \phi_{2}$

$g_{1}=\left(M+M_{2}\right) \times d_{1} \times \cos \phi_{2}+M_{2} \times d_{2} \times \cos \left(\phi_{1}+\phi_{2}\right)$

$g_{2}=M_{2} \times d_{2} \times \cos \left(\phi_{1}+\phi_{2}\right)$

Thus, it can be said that the proposed fuzzy SMC has isolates the unknown parameters in the navigation system. The system has adopted a control law for both low frequency and high-frequency control. In this DCCS system, the robustness is parameterized by considering the fast varying closed loops while the chattering in the system is minimized by using boundary layer (smoothing) technique. The next section highlights the results and analysis of the proposed DCCS mechanism.

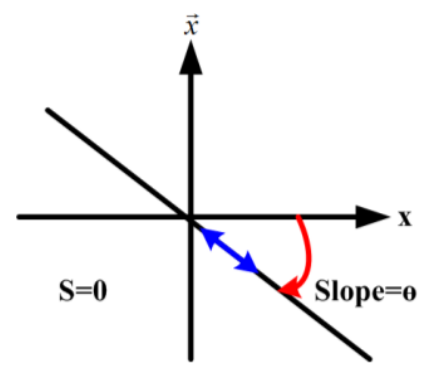

Figure 2. Graphical representation of sliding surface

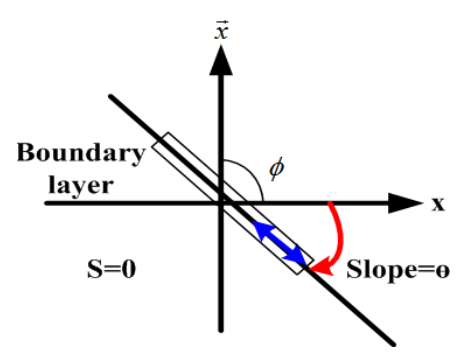

Figure 4. Smoothing in chattering phenomenon

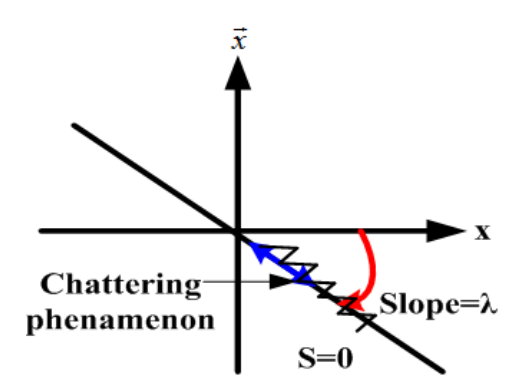

Figure 3. Graphical representation of chattering in control switching

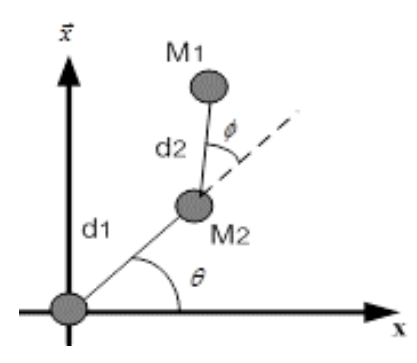

Figure 5. Graphical representation of chattering in control switching

\section{RESULTS AND ANALYSIS}

The system design of the proposed DCCS model is performed by using MATLAB based simulation. The system considers the fuzzy input for cruise control. The performance of the system is analyzed in terms of accuracy in identifying the error and computational complexity. Figure 6 gives the error analysis in DCCS model in terms of tracking error (in radians) Vs. time (in seconds) for both the (a) link 1 and (b) link 2. The simulation is conducted over the DCCS system for $10 \mathrm{sec}$ of time (x-axis). The mass of the links 1,2 is adjusted as $0.75 \mathrm{~kg}$ and $1.25 \mathrm{~kg}$ respectively while the scalar parameter $(\lambda)$ is adjusted to 5 for both the links. Further, the boundary layer thickness is adjusted to 0.02. The outcomes are given in Figure 6 suggests that the tracking of errors in the navigation system is gradually increasing and becomes error free as the time goes in tracking the path. Hence, it can be observed that the in 10 seconds of time, the tracking error becomes zero.

The torque in the navigation system accuracy of the DCCS model. In Figure 7, the torque at link-1 where it can be observed that torque (is represented in the y-axis) against time (in x-axis). Here, the torque variation is intially leading to with better accuracy in navigation and decrement in torque rate is observed due chattering effect. In the navigation system, the speed of the cruise is controlled by the torque. Similarly, Figure 8 indicates the torque variations at link- 2 where the torque is intially leading with better accuracy in navigation and in later part the switching action is taking place in the navigation system. 

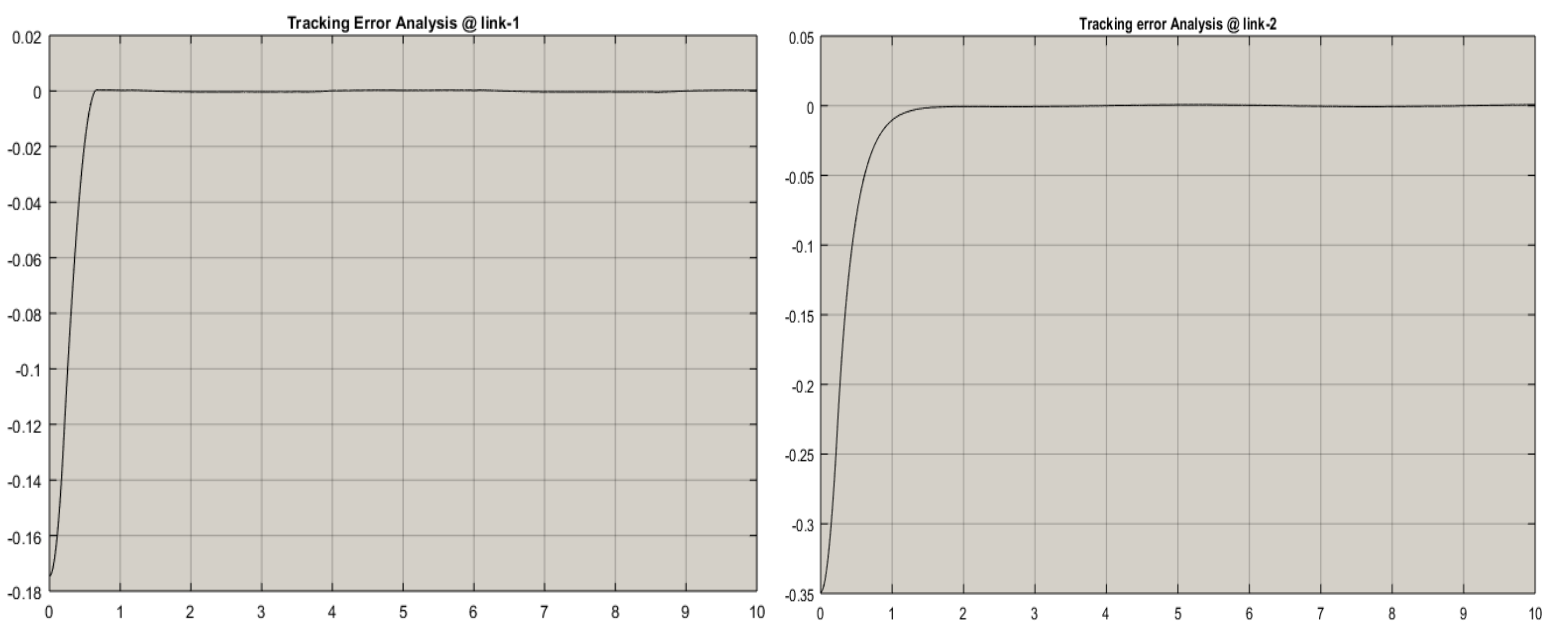

Figure 6. Tracking error analysis at (a) link-1 and (b) link-2

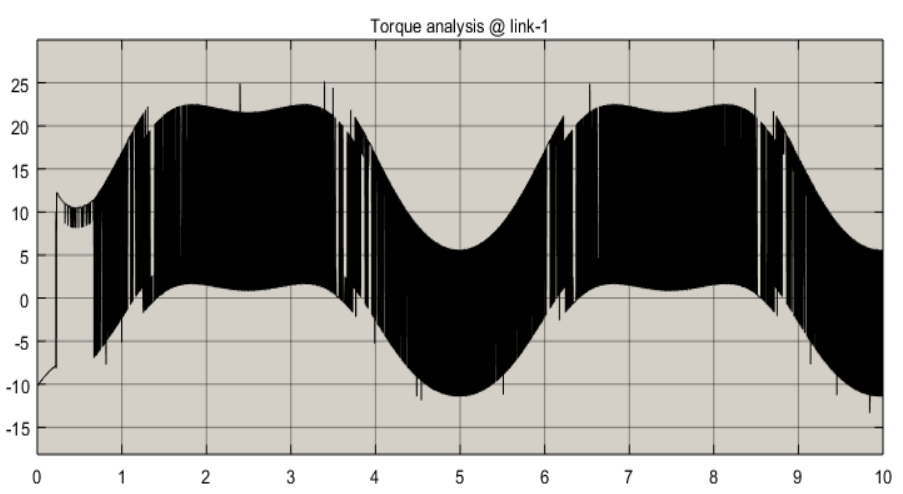

Figure 7. Torque analysis at link-1

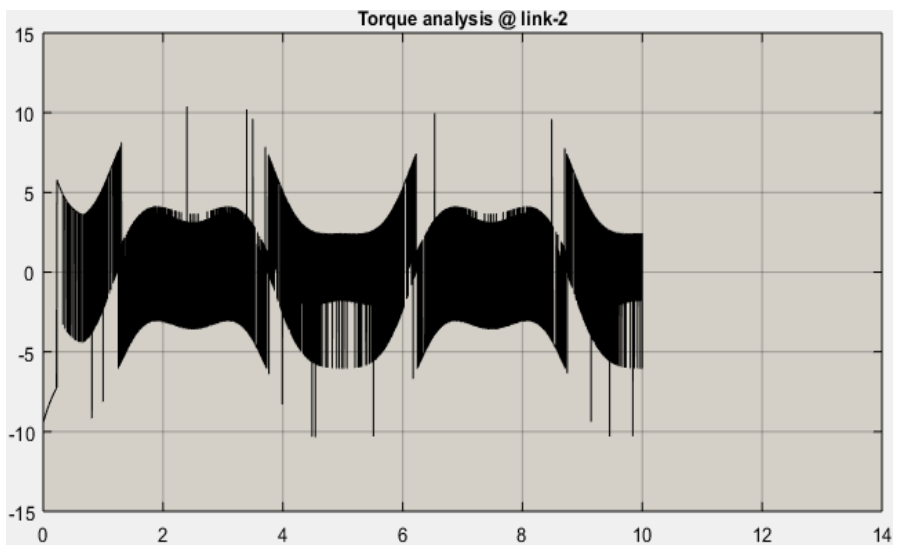

Figure 8. Torque analysis at link-2

In the proposed DCCS model, the boundary layer concept is used to control the navigation system behaviour between link-1 and link-2, where the energy in this boundary layer is linked to the sliding surface of the cruise. This dynamic nature of the proposed system brings effective handling of the speed of the cruise. In the proposed system, the robustness is measure with the reduced chattering in the navigation system, and this is achieved through boundary layer. Further, the computational complexity analysis is performed by considering the time of computation, the clock speed of the navigation system. From the simulation, it is observed that the simulation phase considered only $28 \mathrm{sec}$ and a clock speed of $1801 \mathrm{MHz}$. 


\section{CONCLUSION}

This manuscript presents a DCCS to achieve better navigation under uncertainties. The proposed system incorporates the fuzzy logic and sliding mode technique along with control law. The performance of the system is analyzed by incorporating sliding mode and fuzzy logic and achieves better accuracy in tracking error, computational complexity ( $28 \mathrm{sec}$ of simulation time) under chattering and switching action operation. Thus, the proposed system can handle the dynamic disturbances in the navigation system, and it can handle the current navigation issues in GPS and INS. Further, the proposed system can be incorporated with other machine learning approaches to enhance the performance of the navigation.

\section{REFERENCES}

[1] N. Dey and A. Mukherjee, "Embedded systems and robotics with open source tools," CRC Press, 2018.

[2] D. Dakopoulos and N. G. Bourbakis, "Wearable obstacle avoidance electronic travel aids for blind: a survey," IEEE Transactions on Systems, Man, and Cybernetics, Part C (Applications and Reviews), vol. 40, no. 1, pp. 25-35, 2009.

[3] V. E. Balas, L. C. Jain, and X. Zhao, "Information Technology and Intelligent Transportation Systems," Proceedings of the 2nd International Conference on Information Technology and Intelligent Transportation Systems (ITITS 2017), Xi'an, China, IOS Press, 2017.

[4] G. Cai, J. Dias, and L. Seneviratne, "A survey of small-scale unmanned aerial vehicles: Recent advances and future development trends," Unmanned Systems, vol. 2, no. 02, pp. 175-199, 2014.

[5] G. Hu, et al, "Modified federated Kalman filter for INS/GNSS/CNS integration," in Proceedings of the Institution of Mechanical Engineers, Part G: Journal of Aerospace Engineering, vol. 230, no. 1, pp. 30-44, 2016.

[6] M. Kourogi, et al., "Indoor/outdoor pedestrian navigation with an embedded GPS/RFID/self-contained sensor system," in International conference on artificial reality and telexistence, pp 1310-1321, 2006.

[7] M. Romanovas, R. Ziebold, and L. Lança, "A method for IMU/GNSS/Doppler Velocity Log integration in marine applications," in 2015 International Association of Institutes of Navigation World Congress (IAIN), 2015.

[8] L. Lasmadi, et al., "Inertial navigation for quadrotor using Kalman filter with drift compensation," International Journal of Electrical and Computer Engineering (IJECE), vol. 7, no. 5, pp. 2596-2604, 2017.

[9] S. Nurmaini and B. Tutuko, "Intelligent Robotics Navigation System: Problems, Methods, and Algorithm," International Journal of Electrical and Computer Engineering (IJECE), vol. 7, no. 6, pp. 3711-3726, 2017.

[10] H. V. Pant, "India's Nuclear Doctrine and Command Structure: Implications for India and the World," Comparative Strategy, vol. 24, no. 3, pp. 277-293, 2005.

[11] G. E. Clubb, "The Sensor Irony: How Reliance on Sensor Technology is Limiting Our View of the Battlefield," Master's thesis, National Defense Univ Norfolk Va Joint Advanced Warfighting School, 2010.

[12] D. Stroikos, "China, India in space and the orbit of international society: power, status, and order on the high frontier," Ph.D. Thesis, The London School of Economics and Political Science (LSE), 2016.

[13] M. Shafiee, K. O'Keefe, and G. Lachapelle, "Context-aware adaptive extended Kalman filtering using Wi-Fi signals for GPS navigation," in ION GNSS, Portland, OR, 2011.

[14] Edwards, Christopher, and Sarah Spurgeon, "Sliding mode control: theory and applications," CRC Press, 1998.

[15] A. Manurung, "A Sliding Mode Control for Robot Manipulator," 2010.

[16] L. Dacheng, L. Zhiguo, and Z. Shuai, "Analysis on the influence of the INS precision in an ultra-tightly coupled INS/GPS integration," in 2016 IEEE Information Technology, Networking, Electronic and Automation Control Conference, Chongqing, 2016, pp. 1106-1111.

[17] S. Y. Cho, "IM-filter for INS/GPS-integrated navigation system containing low-cost gyros," IEEE Transactions on Aerospace and Electronic Systems, vol. 50, no. 4, pp. 2619-2629, 2014.

[18] B. P. Duong and V. H. Nguyen, "Development of a GPS/INS integrated navigation system for model aircraft," in 2014 14th International Conference on Control, Automation and Systems (ICCAS 2014), Seoul, 2014, pp. 201-206.

[19] J. Fang and X. Gong, "Predictive Iterated Kalman Filter for INS/GPS Integration and Its Application to SAR Motion Compensation," IEEE Transactions on Instrumentation and Measurement, vol. 59, no. 4, pp. 909-915, 2010.

[20] I. U. Lee, et al., "Navigation system development of the Underwater Vehicles using the GPS / INS sensor fusion," in 2014 14th International Conference on Control, Automation and Systems (ICCAS 2014), Seoul, pp. 610-612, 2014.

[21] Q. Li and F. Sun, "Strong tracking cubature Kalman filter algorithm for GPS/INS Integrated Navigation System," in 2013 IEEE International Conference on Mechatronics and Automation, Takamatsu, pp. 1113-1117, 2013.

[22] Z. Wu, et al., "A filtering algorithm for GPS/INS integrated navigation System based on IMM-AF," in 2016 IEEE International Geoscience and Remote Sensing Symposium (IGARSS), Beijing, pp. 838-841, 2016.

[23] W. Yan, et al., "High accuracy Navigation System using GPS and INS system integration strategy," in 2016 IEEE International Conference on Cyber Technology in Automation, Control, and Intelligent Systems (CYBER), Chengdu, pp. 365-369, 2016.

[24] H. Nakanishi, S. Kanata and T. Sawaragi, "GPS-INS-BARO hybrid navigation system taking into account ground effect for the autonomous unmanned helicopter," in 2012 IEEE International Symposium on Safety, Security, and Rescue Robotics (SSRR), College Station, TX, pp. 1-6, 2012. 
[25] H. Qin, L. Cong and X. Sun, "Accuracy improvement of GPS/MEMS-INS integrated navigation system during GPS signal outage for land vehicle navigation," Journal of Systems Engineering and Electronics, vol. 23, no. 2, pp. 256-264, 2012.

[26] C.-J. Sun, et al., "Implementation of GPS/INS navigation system using low-cost MEMS sensors," in 2010 th IEEE Conference on Industrial Electronics and Applications, Taichung, pp. 1588-1592, 2010.

[27] S. Z. Jamal, "Tightly coupled GPS/INS airborne navigation system," IEEE Aerospace and Electronic Systems Magazine, vol. 27, no. 4, pp. 39-42, 2012.

[28] M. A. K. Jaradat and M. F. Abdel-Hafez, "Nonlinear Autoregressive Delay-Dependent INS/GPS Navigation System Using Neural Networks," IEEE Sensors Journal, vol. 17, no. 4, pp. 1105-1115, 2017.

[29] W. Xin, et al, "Fault detection and diagnosis in the INS/GPS navigation system," in 2014 World Automation Congress (WAC), Waikoloa, HI, pp. 27-32, 2014.

[30] J. Ye, P. Malysz, and A. Emadi, "A Fixed-Switching-Frequency Integral Sliding Mode Current Controller for Switched Reluctance Motor Drives," IEEE Journal of Emerging and Selected Topics in Power Electronics, vol. 3, no. 2, pp. 381-394, 2015.

[31] A. A. A. Elgammal and M. F. El-naggar, "MOPSO-based optimal control of shunt active power filter using a variable structure fuzzy logic sliding mode controller for hybrid (FC-PV-Wind-Battery) energy utilisation scheme," IET Renewable Power Generation, vol. 11, no. 8, pp. 1148-1156, 2016.

[32] J. Fei, Y. The Chu and S. Hou, "A Backstepping Neural Global Sliding Mode Control Using Fuzzy Approximator for Three-Phase Active Power Filter," in IEEE Access, vol. 5, pp. 16021-16032, 2017.

[33] H. Li, et al., "Optimal Guaranteed Cost Sliding-Mode Control of Interval Type-2 Fuzzy Time-Delay Systems," IEEE Transactions on Fuzzy Systems, vol. 26, no. 1, pp. 246-257, 2018.

[34] Q. Shen, P. Shi and Y. Shi, "Distributed Adaptive Fuzzy Control for Nonlinear Multiagent Systems Via Sliding Mode Observers," IEEE Transactions on Cybernetics, vol. 46, no. 12, pp. 3086-3097, 2016.

[35] T. Wang and J. Fei, "Adaptive Neural Control of Active Power Filter Using Fuzzy Sliding Mode Controller," IEEE Access, vol. 4, pp. 6816-6822, 2016.

[36] S. Wen, M. Z. Q. Chen, Z. Zeng, X. Yu and T. Huang, "Fuzzy Control for Uncertain Vehicle Active Suspension Systems via Dynamic Sliding-Mode Approach," IEEE Transactions on Systems, Man, and Cybernetics: Systems, vol. 47, no. 1, pp. 24-32, 2017.

[37] S. Wen, M. Z. Q. Chen, Z. Zeng, T. Huang, and C. Li, "Adaptive Neural-Fuzzy Sliding-Mode Fault-Tolerant Control for Uncertain Nonlinear Systems," IEEE Transactions on Systems, Man, and Cybernetics: Systems, vol. 47, no. 8, pp. 2268-2278, 2017.

[38] M. Habbab, A. Hazzab, and P. Sicard, "Real Time Implementation of Fuzzy Adaptive PI-sliding Mode Controller for Induction Machine Control," International Journal of Electrical and Computer Engineering (IJECE), vol. 8, no. 5, pp. 2883-2893, 2018.

[39] X. Zhao, et al., "Adaptive Fuzzy Hierarchical Sliding-Mode Control for a Class of MIMO Nonlinear Time-Delay Systems with Input Saturation,” IEEE Transactions on Fuzzy Systems, vol. 25, no. 5, pp. 1062-1077, 2017.

[40] T. Someswari, A. K. Tiwari, and R. Nagaraj, "Probing the efficacy of research contribution towards fuzzy sliding mode controller," in 2017 International Conference on Circuits, Controls, and Communications (CCUBE), 2017.

[41] T. Someswari and A. K. Tiwari, "Development of Sliding Mode Fuzzy Controller for Advanced Navigation System," Development, vol. 6, no. 1, 2017.

[42] X. Li and W. Zhang, "An adaptive fault-tolerant multisensor navigation strategy for automated vehicles," IEEE Transactions on Vehicular Technology, vol. 59, no. 6, pp. 2815-2829, 2010.

[43] F. Yang, et al., "Practical integrated navigation fault detection algorithm based on sequential hypothesis testing," Journal of Systems Engineering and Electronics, vol. 22, no. 1, pp. 146-149, 2011.

[44] D. Li, et al., "Fault tolerant navigation method for satellite based on information fusion and unscented Kalman filter," Journal of Systems Engineering and Electronics, vol. 21, no. 4, pp. 682-687, 2010.

[45] A. Valizadeh, and H. Hamidi, "Improvement of navigation accuracy using tightly coupled kalman filter," International Journal of Engineering, vol. 30, no. 2, pp. 215-223, 2017.

[46] T. Someswari, A. K. Tiwari, and R. Nagaraj, "Novel framework for navigation using the enhanced fuzzy approach with sliding mode controller," International Journal of Electrical and Computer Engineering, vol. 8, no. 6, pp. 4310-4320, Dec. 2018.

\section{BIOGRAPHIES OF AUTHORS}

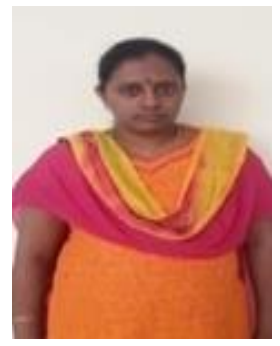

T. Someswari working as assistant professor, EEEdept at The Oxford college of Engineering has published 3 interantional journal papers. I am pursuing Ph.D in VTU under the guidance of Dr. Anilkumar and Dr. Nagaraj R Ihave completed my B.E. degree in 2007 from JNTU, Hydearbad in Electrical and Electroincs and M.E. from JNTU, Anantapur in 2010. 


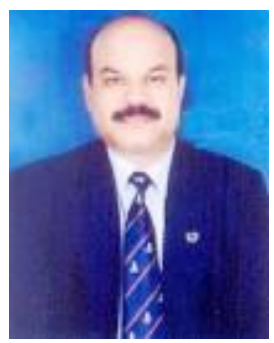

Dr. Anilkumar Tiwari has total of 26 years of experience in Industry, research and teaching (UG \& PG) students. I have been worked as Professor \& HOD, Department of ECE in The Oxford College of Engineering, Bangalore Jun 2011. Served as Senior PG faculty in Air Force Technical College, Bangalore Jun 2009-May 2011. Served in Indian Air Force as Senior Engineer super specialising in the areas of Microwave and Radar Engg and Satellite \& Digital Communication, Advanced Missile Guidance and Control Research assignment at Defence Institute of advanced Technology and Defence services staff college.Presently working as Director, Amity School of Engineering.

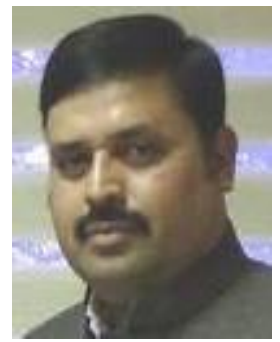

Dr. Nagaraj $\mathbf{R}$ has completed his Ph.D degree from vtu and has published various international and national journals.He has taken his Masters degree from Gulbarga university and B.E from Bangalore university.He has worked as a Director, Dhirubai institute of sciences and The Oxford college of engineering. Presently, He is working as a vice chancellor, Kalasalingam University, krishnankoil, Madurai, India 